Théologiques

Théologiques

\title{
Comprendre la superstition
}

\section{Jean-Claude Breton}

Volume 8, numéro 1, printemps 2000

La superstition

URI : https://id.erudit.org/iderudit/005002ar

DOI : https://doi.org/10.7202/005002ar

Aller au sommaire du numéro

Éditeur(s)

Faculté de théologie de l'Université de Montréal

ISSN

1188-7109 (imprimé)

1492-1413 (numérique)

Découvrir la revue

Citer cet article

Breton, J.-C. (2000). Comprendre la superstition. Théologiques, 8(1), 9-17.

https://doi.org/10.7202/005002ar

\section{Résumé de l'article}

La superstition a acquis une nouvelle signification dans le mondeinterreligieux dans lequel nous vivons, un monde qui est passablementdifférent de celui de l'ère chrétienne homogène. Elle est aussi étudiée demanière différente. Plutôt que d'être représentée seulement comme une voiereligieuse déviante, elle est comprise de plus en plus comme un monde desens que la science, pas plus que la religion, ne peut atteindre par elle-même.Cette approche positive de la superstition n'est pas sans poser de nouvellesquestions, l'une étant la difficulté de faire cohabiter sympathie, ouverture etapproche critique. Ce bref article essaie de présenter un survol de lasituation présente. d'utilisation que vous pouvez consulter en ligne.

https://apropos.erudit.org/fr/usagers/politique-dutilisation/ 


\section{Comprendre la superstition}

Jean-Claude BRETON

Faculté de théologie

Université de Montréal

«Es-tu superstitieux? » Cette formule, peut-être impolie aux yeux de certains, est quand même doublement révélatrice du caractère particulier du sujet que nous abordons ici. Elle nous dit d'abord le degré d'intimité nécessaire pour qu'il soit possible d'oser demander à quelqu'un s'il est superstitieux et surtout d'espérer une réponse. Plus profondément encore, cette question apparemment intrusive indique un déplacement majeur observable dans notre milieu depuis quelques décennies.

Au moment où le Québec vivait dans l'unanimité religieuse, il n'était pas très compromettant de répondre à cette question, car la réponse se situait toujours sur un fond de foi catholique officielle. De fait, la question signifiait alors : "Fais-tu une place, plus ou moins grande, à des pratiques superstitieuses dans ton adhésion à la foi catholique? » Il valait évidement mieux s'abstenir de gestes superstitieux, mais en pratiquer quelques-uns ne disqualifiait pas pour autant un croyant. L'opinion populaire, plus que le discours des élites religieuses mieux informées, accordait facilement un certificat de "bonne foi » à une personne qui avouait par ailleurs craindre les chats noirs, ne pas vouloir passer sous une échelle et surtout refuser de « faire une croix » en donnant la main.

On garde encore aujourd'hui un reste de cette attitude d'autrefois quand, par exemple, on évoque certaines pratiques des vedettes du sport. Le contexte donne à comprendre qu'on renvoie alors à un rituel personnel, étrange et irrationnel plutôt qu'à la foi de ces idoles. Mais une nouvelle situation tend à s'installer dans le discours sur la superstition. Demander à quelqu'un s'il est superstitieux est en voie de devenir une question directement posée à la foi qu'il investit, ou non, dans tel objet ou telle pratique. Face au nouvel âgiste, qui lui parle 
chakras et cristaux, le chrétien peut en venir à soulever la question : "Crois-tu vraiment en cela? » Quitte à se faire renvoyer des questions du même genre à propos de ses dévotions, de ses images et de ses statues. L'option religieuse de l'autre tend à devenir la nouvelle superstition.

La superstition n'est plus seulement un risque de contamination plus ou moins grave d'une foi par ailleurs orthodoxe et tenue dans la fidélité ; elle devient le nom donné à des pratiques religieuses différentes, étrangères, autres que les siennes. Demander à quelqu'un s'il est superstitieux devient de plus en plus délicat, car ce n'est plus le simple fait d'évaluer la qualité de sa foi et de ses pratiques ; c'est devenu une sorte de soupçon sur leur qualité et leur authenticité. En cela, l'évolution observable dans les comportements majoritaires chez nous illustre aussi les chemins pris par les discours savants dans leurs rapports à la superstition. Là aussi, il y a déplacement du discours qui passe de la simple affirmation au soupçon, puis de l'examen systématique au questionnement réciproque. Le discours savant a aussi son histoire.

\section{Évolution du discours savant ${ }^{1}$}

L'étymologie du mot est déjà indicative. La superstition est ce qui reste, ce qui survit, ce qui dépasse en somme des mœurs anciennes, ou des mœurs autorisées et légalement acceptées. Thomas d'Aquin suggérait un sens proche quand il en parlait comme d'un excès de la vertu de religion $;^{2}$ il y avait pour lui aussi quelque chose de trop dans la superstition. Mais ces commentaires se situent toujours en contexte de chrétienté et sont formulés en termes théologiques.

1. Il n'est pas possible de fournir ici une bibliographie complète sur le sujet, mais on pourra s'initier à la problématique en consultant des ouvrages comme : Françoise ASKEVIS-LEHERPEUX, La superstition (Que sais-je? 2370), Paris, PUF, 1988, 127 p., et deux articles dans Isaac CHIVA et Utz JEGGLE (dir.) Ethnologies en miroir. La France et les pays de langue allemande, Paris, Éd. de la Maison des sciences de l'homme, 1987, 396 p., soit Élisabeth CLAVERIE, "Les symbolismes majeurs, p. 139-149 et FAVREVASSAS et D. FAVRE, "L'ethnologie du symbolisme en France », p. 123138 ; voir aussi Masimo INTROVIGNE, La magie à nos portes, Montréal, Fides, 1994, 119 p.

2. L'autre excès identifié étant l'irreligion. Voir Summ. theol. II-II, q 92 et 94. 
Faute de proposer une histoire même sommaire de la superstition, j'évoquerai ici quelques études qui montrent son existence avant le christianisme et sa permanence même après l'apparition de celui-ci. Chez les Grecs, ${ }^{3}$ mais aussi dans toute la culture gréco-romaine, ${ }^{4}$ une place est faite à ce que la science appelle pudiquement la magie. Pudeur qu'on retrouvera encore à propos des populations du Nouveau Testament comme à Éphèse et dans les écrits de l'évangéliste Luc. ${ }^{5}$ Un évêque français, Césaire d'Arles, s'est aussi intéressé à la question qui porte maintenant le nom de superstition, ${ }^{6}$ déjà présent chez Augustin. ${ }^{7}$ D'aussi bonnes racines n'étaient pas préludes à une mort rapide. La superstition, encore parfois nommée sorcellerie, se maintient au Moyen Âge chrétien. ${ }^{8}$ De fait, la superstition court à travers les âges, ${ }^{9}$ et pas seulement en contexte français. ${ }^{10} \mathrm{D}$ 'ailleurs elle va aussi survivre aux efforts de puri-

3. Voir E.R. DODDS, Les Grecs et l'irrationnel, Berkeley, University of California Press, 1959, 317 p.

4. Voir Fritz GRAF, La magie dans l'antiquité gréco-romaine. Idéologie et pratique, Paris, Les Belles Lettres, 1994, 322 p. Encore André NEYTON, Le merveilleux religieux dans l'Antiquité. Aspects choisis, Paris, Letouzey et Ané éditeurs, 1991, $128 \mathrm{p}$.

5. Voir Clinto E. ARNOLD, Ephesians: Power and Magic. The Concept of Power in Ephesians in Light of its Historical Setting, Cambridge, Cambrige University Press, 1989, 244 p. Ou encore Susan R. GARRET, Magic and the Demonic in Luke's Writings, Minneapolis, Fortress Press, 1989, 179 p.

6. Voir Guillaume KONDA, Le discernement et la malice des pratiques superstitieuses d'après les sermons de S. Césaire d'Arles, Rome, Officium Libri Catholici/Catholic Book Agency, 1970, 58 p.

7. S. Augustin a une note dans La cité de Dieu, (Oeuvres de S. Augustin, t. 33 ) DDB, 1959, p. 813-814, qui rappelle qu'après son avènement, le christianisme fut désigné par le mot superstition par des auteurs comme Pline le Jeune, Tacite et Suétone!

8. Voir D.D. McCULLOCH, Medieval Faith and Fable, London, George G. Harrap and Company Ltd, 1932, 345 p. Aussi Robert MUCHEMBLED (dir.), Magie et sorcellerie en Europe. Du Moyen Âge à nos jours, Paris, Armand Collin, 1994, $435 \mathrm{p}$.

9. Voir Andrée RUFFAT, La superstition à travers les âges, Paris, Payot, 1977, $297 \mathrm{p}$.

10. Voir Marie-Sylvie DUPONT-BOUCHAT, «Sorcellerie et superstition : l'attitude de l'Église dans les Pays Bas (XVI $-\mathrm{XVIII}{ }^{\mathrm{e}}$ siècles)» dans : Hervé HASQUIN, Magie, sorcellerie, parapsychologie, Bruxelles, Éditions de l’Université de Bruxelles, 1984, 240, p. 61-83. 
fication de la révolution, ${ }^{11}$ de sorte qu'il est encore possible d'en relever les traces dans la paysannerie française du 19eme siècle. ${ }^{12}$

En somme, une étude historique plus poussée montrerait que le discours sur la superstition a pour ainsi dire préparé et accompagné l'apparition, l'expansion et le développement du christianisme en occident. La situation va connaître des changements importants suite à la mise en contact avec des sociétés humaines différentes et l'apparition des sciences humaines. La découverte de continents inconnus et de leurs populations fournira l'occasion de recherches et de réflexions inédites parce qu'impossibles en contexte de chrétienté.

Les écoles anglaise et française ${ }^{13}$ d'ethnologie vont tenter de trouver une signification aux faits observés dans les colonies et aussi dans certaines pratiques régionales. ${ }^{14} \mathrm{Au}$ début, la superstition est comprise surtout en opposition à la religion et plus précisément à la religion connue de l'observateur. Comme dans le religion sous sa forme la plus communément éprouvée en occident, la superstition se revêt de prétentions sotériologiques. Si la religion chrétienne offre un salut venu de Dieu et offert gratuitement à qui l'accueille de façon convenable, la superstition propose des rituels porteurs de salut à qui les pratique dans le respect des règles et des interdits. ${ }^{15}$

11. Voir Éloïse MOZZANI, Magie et superstitions : de la fin de l'Ancien Régime à la Restauration, Paris, Éditions Robert Laffont, 1988, 462 p. Comme en témoigne aussi la réaction de l'abbé Thiers : voir Jean-Marie GOULEMOT, "L'Autre du préjugé. Le Traité des superstitions de l'abbé Thiers ", dans Le temps de la Réflexion, V (1984) p. 214.

12. Judith DEVLIN, The Superstitious Mind. French Peasants and the Supernatural in the Nineteenth Centuty, New Haven/London, Yale University Press, 1987 (1952), 316 p.

13. On pense ici aux classiques que sont devenus : Frazer, Hubert, Mauss, Malinowski et Evans-Pritchard.

14. On pourra consulter, par exemple, J.G. FRAZER, La tâche de Psyché. De l'influence de la superstition sur le développement des institutions, Paris, Librairie Armand Colin, 1914, 298 p. Et plus près de chez nous : Fernand DUMONT, Jean-Paul MONTMINY, Michel STEIN et al., Le merveilleux, Québec, Les Presses de l'Université Laval, 1973, 163 p.

15. Pour trouver une définition de la superstition autour du tryptique magie, sorcellerie, divination et connaître la lutte de l'Église contre la superstition, voir Jean MELLOT, La superstition, ersatz de la foi, Paris, Arthème Fayard, 1959, 120 p. 
À mesure que les sciences humaines vont se développer et perfectionner leurs méthodes, elles nuanceront leurs commentaires et leurs explications. La sociologie et la psychologie ${ }^{16}$ mèneront des enquêtes sur des groupes et des individus pour mieux savoir ce qui se fait vraiment au nom de la superstition et pour tâcher de comprendre de quoi il retourne. Deux approches théoriques sont repérables presque tout au long du développement de ces recherches. D'un côté, la dimension jugée déviante des pratiques marginales proposées par les différentes superstitions fait naître une intervention thérapeutique. On diagnostique ce qui ne fonctionne pas et on s'efforce de trouver un correctif qui servira de remède adéquat. De l'autre, la réaction insiste moins sur l'aspect déviant, anormal ou maladif, pour s'attacher plutôt à reconnaître une différence, une anomalie ou un écart susceptible d'ouvrir sur un sens nouveau. La préoccupation sémiotique prend le dessus et l'effort consiste à décoder ce qui au départ ne semble que hiéroglyphes.

En lien avec ces différentes interprétations, une constante s'est imposée : la nécessité d'observer de façon aussi précise et exhaustive que possible la réalité à comprendre. Des traces des observations factuelles sont présentées sur de nombreux sites du réseau Internet. Leur consultation renseigne sur les gestes, les dictons, les maximes et autres qui ont pu être répertoriés dans différents pays ou régions du monde. Plus on pousse ces observations, plus la question s'impose toutefois de savoir pourquoi parler de superstition dans ces cas et pas dans celui des religions officielles? Sans compter que ces observations permettent aussi de comprendre que la superstition se comprend comme un phénomène d'opposition non seulement à la religion, mais aussi à la science.

Le phénomène d'oposition à la science est contemporain. Il semble en effet que les progrès de la science se sont accompagnés de pratiques parallèles qu'on en est venu à appeler aussi superstitieuses. ${ }^{17}$ Plutôt que du côté du salut, c'est en parallèle avec le savoir certain et

16. Voir, par exemple, Conrad ZUCKER, Psychologie de la superstition, Paris, Payot, 1972, 242 p. et Léonard ZUZNE et Jones WARREN, Anomalistic Psychology. A Study of Magical Thinking, London, Lawrence Erlbaum Associates Publishers, 1989, 316 p.

17. Voir à ce propos: René ALLEAU, Histoire des sciences occultes, Genève, Édito-Service S.A., 1965, 112 p. 
vrai de la science que la superstition va se développer. Là où les savoirs scientifiques essaient de relever une séquence causale rationnelle pour expliquer les phénomènes observés, la superstition ne se gênera pas d'offrir des explications s'appuyant sur une lecture irrationnelle de la causalité des événements ; lecture irrationnelle le plus souvent camouflée sous des emprunts effectués dans le langage scientifique. À titre d'exemples, on peut penser ici aux explications proposées pour continuer de justifier une vision géocentrique de l'univers ou pour " prouver » l'existence des OVNI et des phénomènes psy. De façon plus personnelle, j'évoquerai un volume reçu en cadeau, qui m'était sans doute offert en raison de mon intérêt pour la vie spirituelle. Il s'agit du volume de Texe Marrs ${ }^{18}$ qui, avant de se lancer dans la dénonciation du projet L.U.C. I.D, s'est mérité ses lettres de créance comme officier dans l'aviation américaine (U. S. Air Force) et comme professeur à l'Université de Austin, Texas. Maintenant, il veut mettre le monde en garde contre la menace d'une prise de contrôle universel de la technologie moderne, aussi identifiée à partir du chiffre 666 de la bête de l'Apocalypse!

Il y a donc un mouvement d'opposition qui s'est peu à peu affirmé, tant du point de vue religieux que du côté de la science, contre la superstition et les pratiques superstitieuses. Un mouvement qui tend à se transformer en approche intégrative à mesure que les chercheurs changent leur regard sur eux-mêmes et leurs façons d'aborder des phénomènes d'abord jugés étranges. Parce que les recherches menées tant au nom du savoir religieux que de celui des autres sciences ont contribué à faire prendre conscience et à révéler que l'opposition identifiée entre le savoir commun, et communément accepté, et celui de la superstition en est un de légitimité et de pouvoir et que son fondement n'est pas toujours aussi évident qu'il pourrait le paraître au premier regard. Il ne s'agit pas bien sûr d'accepter tous les discours fantaisistes élaborés en marge du religieux et du scientifique, mais de reconnaître qu'un certain nombre de ces discours sont disqualifiés pour des raisons obscures. En dernière analyse, l'examen de la superstition renvoie à la question de savoir au nom de quoi, ou de qui, se fonde l'ailleurs qui prétend l'évaluer et la critiquer.

18. Texe MARRS, Project L.U.C.I.D. The Beast 666 Universal Human Control System, Austin Texas, Living Truth Publishers, 1996, 224 p. 
Dans la mesure où les pratiques superstitieuses sont des témoins, des restes de pratiques religieuses ou scientifiques anciennes, ne peuvent-elles pas être porteuses de références et de valeurs à prendre en compte. Il n'irait donc plus de soi d'accoler une connotation péjorative à l'emploi du mot superstition. "Étymologique, nous dit Nicole Belmont, la superstition ne s'oppose pas à la religion, puisqu'elle est à proprement parler une survivance, sans qu'il ne s'attache rien de péjoratif à cette notion : témoin qui a survécu à un état de choses passé, monument qui le rappelle. ${ }^{19} \mathrm{La}$ superstition peut donc aussi être entrevue comme faisant partie, dans l'univers socio-culturel, d'un rapport de coexistence et de tolérance où circule non pas des « débris de civilisations mortes " comme l'entendait l'école anthropologique anglaise de Edward Burnett Tylor, ${ }^{20}$ mais comme des réseaux de sens et d'association qui persistent et évoluent dans la cité.

\section{Oui, mais...}

Ceci dit, la question se profile alors de savoir si l'on n'est pas en train de verser dans une nouvelle superstition. Abandonner toute démarche critique à l'égard de la superstition n'équivaut-il pas à emprunter un chemin en tout semblable à celui traditionnellement appelé superstitieux? Si le discours officiel enlève à la superstition, surtout religieuse, son caractère négatif, ne faut-il pas anticiper la naissance éventuelle d'un nouveau discours superstitieux, sorte de nouveau "reste ", qui vient interroger cette attitude? En mots clairs : est-ce rationnel et acceptable de ne plus se méfier de la superstition ou s'agit-il là d'une nouvelle superstition, d'un produit, adapté à la modernité, du comportement superstitieux le plus ancien?

Car si la superstition constitue un reste, un témoin, d'une époque passée, il est légitime de penser que le déplacement qui l'a produite était favorisé et porté par une certaine légitimité. Les déplacements dans les manières de croire aussi bien que ceux imposés par le développement des sciences ont leurs raisons d'être. Si on ne croit plus de

19. Nicole BELMONT, "Superstition et religion populaire dans les sociétés occidentales » dans Michel IZARD et Pierre SMITH (dir.), La fonction symbolique, (NRF, Bibliothèque des sciences humaines), Paris, Gallimard, 1979, p. 66.

20. Ibid. p. 55. 
la même façon et si on confie de plus en plus de questions au savoir scientifique, ce n'est pas sans conséquence pour ce qui reste des convictions anciennes. Ces reliquats peuvent bien donner à comprendre de grands pans de l'histoire. Faut-il pour autant emprunter à leur logique pour aménager le présent?

\section{Enjeux de la recherche}

Dès lors que cesse d'exister une opposition convenue, qui servait à démoniser toute superstition, la question de son existence et de son sens s'impose avec encore plus d'acuité. Pourquoi la superstition existe-t-elle, à quoi sert-elle, quel est son rôle dans la vie sociale et individuelle? Ultimement, mais peut-être aussi en raison de ses relations originales avec la religion et la science, la question resurgit de l'apport de la superstition à la recherche du vrai. Dans l'état actuel des réflexions théologiques et épistémologiques, tenant compte surtout de la place que ces réflexions ont été amenées à faire aux mythes, aussi bien en religion qu'en science, l'heure n'est-elle pas venue de se demander quel est le sens des mythes engendrés par la superstition?

Si la religion et la science semblent parfois être parvenues à rendre compte, au moins en partie, des fondements de leur discours respectif, il n'en reste pas moins que le vertige envahit parfois aussi les esprits tant des théologiens que des scientifiques. Le paradoxe n'en ressort que plus grand et plus fort. Comment se fait-il que notre âge du savoir scientifique absolu parvenu à une telle vision critique qu'il consent parfois à reconnaître la fragilité de certains de ses points d'appui traditionnels, s'avère fermé et intolérant aux propos du discours qu'il qualifie de superstitieux? Peut-être est-ce justement en raison du ferme propos du discours superstitieux de ne pas se livrer à un examen critique de lui-même et de ses fondements? À la limite la difficulté, sinon l'impossibilité, du dialogue entre la superstion et la religion ou la science ne vient-il pas du refus de celles-ci de se soumettre à une remise en cause radicale? Mais faut-il que tous les discours aient subit l'épreuve de la critique? N'est-il plus possible dans notre monde de parler simplement pour exprimer ses émotions, ses sensations et même ses désirs sans autre souci que de les dire?

S'il est impossible de disqualifier la superstition au nom de l'absolu du savoir religieux ou scientifique communément accepté, sauf à commettre une sorte d'abus de pouvoir, ne s'impose-t-il pas de 
se demander quelle est la contribution positive de cet autre discours que je ne m'autorise plus à renvoyer aux gémonies?

\section{RÉSUMÉ}

La superstition a acquis une nouvelle signification dans le monde interreligieux dans lequel nous vivons, un monde qui est passablement différent de celui de l'ère chrétienne homogène. Elle est aussi étudiée de manière différente. Plutôt que d'être représentée seulement comme une voie religieuse déviante, elle est comprise de plus en plus comme un monde de sens que la science, pas plus que la religion, ne peut atteindre par elle-même. Cette approche positive de la superstition n'est pas sans poser de nouvelles questions, l'une étant la difficulté de faire cohabiter sympathie, ouverture et approche critique. Ce bref article essaie de présenter un survol de la situation présente.

\section{ABSTRACT}

Superstition has gained a new signification in the interreligious world we live in, which is quite different from the one it had during the homogeneous christian era. It is also studied differently. Rather than being sketched mainly as a devious religious way, it is understood more and more as a world of meaning which science, as well as religion, is not always able to reach by itself. This positive approach to superstition is however not without opening new questions, one being the difficulty to mix sympathy and openness with a critical appraisal. This paper tries to offer an overview of the present situation. 